\title{
Search for neutrino emission from gamma-ray flaring blazars with the ANTARES telescope
}

\section{S. Adrián-Martínez ${ }^{\text {a }}$, I. Al Samarai ${ }^{\mathrm{b}}$, A. Albert $^{\mathrm{c}}$, M. Andréd ${ }^{\mathrm{d}}$, M. Anghinolfi ${ }^{\mathrm{e}}$, G. Anton $^{\mathrm{f}}, \mathrm{S}$. Anvar ${ }^{\mathrm{g}}$, M. Ardid ${ }^{\mathrm{a}}$, T. Astraatmadja ${ }^{\mathrm{h}, 1}$, J-J. Aubert ${ }^{\mathrm{b}}$, B. Baret ${ }^{\mathrm{i}}$, S. Basa ${ }^{\mathrm{j}}$, V. Bertin $^{\mathrm{b}}$, S. Biagi ${ }^{\mathrm{k}, \mathrm{l}}$, C. Bigongiari ${ }^{\mathrm{n}}$, C. Bogazzi $^{\text {h }}$, M. Bou-Cabo ${ }^{a}$, B. Bouhou ${ }^{i}$, M.C. Bouwhuis ${ }^{\text {h }}$, J. Brunner ${ }^{b, 2}$, J. Busto ${ }^{b}$, F. Camarena $^{a}$} A. Capone ${ }^{\mathrm{o}, \mathrm{p}}$, C. Cârloganu ${ }^{\mathrm{q}}$, G. Carminati ${ }^{\mathrm{k}, \mathrm{l}, 3}$, J. Carr $^{\mathrm{b}}$, S. Cecchini ${ }^{\mathrm{k}}$, Z. Charif $^{\mathrm{b}}$, Ph. Charvis ${ }^{\mathrm{r}}$, T. Chiarusi ${ }^{\mathrm{k}}$, M. Circella ${ }^{\mathrm{s}}$, L. Core ${ }^{\mathrm{b}}$, H. Costantini ${ }^{\mathrm{b}}$, P. Coyle ${ }^{\mathrm{b}}$, A. Creusot ${ }^{\mathrm{i}}$, C. Curtil $^{\mathrm{b}}$, G. De Bonis ${ }^{\text {o,p }}$, M.P. Decowski ${ }^{\mathrm{h}}$, I. Dekeyser ${ }^{t}$, A. Deschamps ${ }^{r}$, C. Distefano ${ }^{u}$, C. Donzaud ${ }^{i, v}$, D. Dornic ${ }^{\text {b,n,* }}$, Q. Dorosti $^{\text {w }}$, D. Drouhin ${ }^{\text {, }}$, T. Eberl ${ }^{f}$, U. Emanuele ${ }^{n}$, A. Enzenhöfer ${ }^{f}$, J-P. Ernenwein ${ }^{b}$, S. Escoffier ${ }^{b}$, K. Fehn ${ }^{f}$, P. Fermani ${ }^{\text {o,p }}$, M. Ferri $^{a}$, S. Ferry ${ }^{\mathrm{x}}$, V. Flaminio ${ }^{\mathrm{m}, \mathrm{y}}$, F. Folger ${ }^{\mathrm{f}}$, U. Fritsch ${ }^{\mathrm{f}}$, J-L. Fuda ${ }^{\mathrm{t}}$, S. Galatà ${ }^{\mathrm{b}}$, P. Gay ${ }^{\mathrm{q}}$, K. Geyer ${ }^{\mathrm{f}}$, G. Giacomelli ${ }^{\mathrm{k}, \mathrm{l}}$, V. Giordano ${ }^{\text {u, J.P. Gómez-González }}{ }^{\mathrm{n}}$, K. Graf ${ }^{\mathrm{f}}$, G. Guillard ${ }^{\mathrm{q}}$, G. Halladjian ${ }^{\mathrm{b}}$, G. Hallewell ${ }^{\mathrm{b}}$, H. van Haren ${ }^{\mathrm{z}}$, J. Hartman ${ }^{\text {h }}$, A.J. Heijboer ${ }^{\text {h }}$, Y. Hello ${ }^{\text {r }}$, J.J. Hernández-Rey ${ }^{\text {}}$, B. Herold ${ }^{\mathrm{f}}$, J. Hößl ${ }^{\mathrm{f}}$, C.C. Hsu ${ }^{\text {h }}$, M. de Jong ${ }^{\text {h,1 }}$, M. Kadler aa, O. Kalekin ${ }^{\text {f }}$, A. Kappes ${ }^{f}$, U. Katz ${ }^{\text {f }}$, O. Kavatsyuk ${ }^{\text {w }}$, P. Kooijman ${ }^{\text {h,ab,ac }}$, C. Kopper ${ }^{\text {h,f }}$

A. Kouchner ${ }^{\mathrm{i}}$, I. Kreykenbohm ${ }^{\text {aa }}$, V. Kulikovskiy ${ }^{\text {ad,e }}$, R. Lahmann ${ }^{\text {f }}$, G. Lambard ${ }^{\text {n }}$, G. Larosa ${ }^{a}$, D. Lattuada ${ }^{\text {, }}$, D. Lefèvre ${ }^{t}$, G. Lim ${ }^{\text {h,ac }}$, D. Lo Presti ${ }^{\text {ae,af }}$, H. Loehner ${ }^{w}$, S. Loucatos ${ }^{x}$, F. Louis ${ }^{g}$, S. Mangano ${ }^{n}$, M. Marcelin ${ }^{j}$,

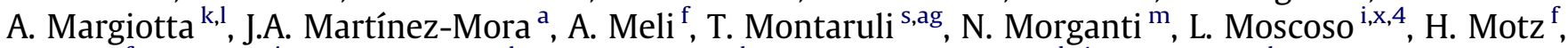

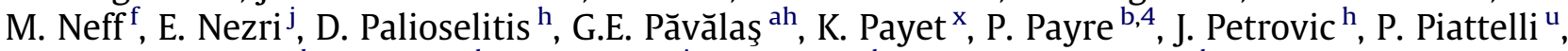
N. Picot-Clemente ${ }^{b}$, V. Popa ${ }^{\text {ah }}$, T. Pradier ${ }^{a i}$, E. Presani $^{\text {h }}$, C. Racca ${ }^{c}$, C. Reed ${ }^{\text {h }}$, G. Riccobene ${ }^{\mathrm{u}}$, C. Richardt $^{\mathrm{f}}$, R. Richter ${ }^{\mathrm{f}}$, C. Rivière ${ }^{\mathrm{b}}$, A. Robert $^{\mathrm{t}}$, K. Roensch $^{\mathrm{f}}$, A. Rostovtsev $^{\mathrm{aj}}$, J. Ruiz-Rivas ${ }^{\mathrm{n}}$, M. Rujoiu ${ }^{\text {ah }}$, G.V. Russo ae,af, F. Salesa ${ }^{\text {n, D.F.E. Samtleben }}{ }^{\text {h }}$, P. Sapienza ${ }^{\mathrm{u}}$, F. Schöck ${ }^{\mathrm{f}}$, J-P. Schuller $^{\mathrm{x}}$, F. Schüssler ${ }^{\mathrm{x}}$, T. Seitz ${ }^{f}$, R. Shanidze ${ }^{f}$, F. Simeone ${ }^{o, p}$, A. Spies ${ }^{f}$, M. Spurio ${ }^{\text {k,l }}$, J.J.M. Steijger ${ }^{\text {h }}$, Th. Stolarczyk ${ }^{\mathrm{x}}$, A. Sánchez-Losa ${ }^{\mathrm{n}}$, M. Taiuti ${ }^{\mathrm{e}, \mathrm{ak}}$, C. Tamburini ${ }^{\mathrm{t}}, \mathrm{S}$. Toscano ${ }^{\mathrm{n}}, \mathrm{B}$. Vallage ${ }^{\mathrm{x}}$, C. Vallée $^{\mathrm{b}}$, V. Van Elewyck ${ }^{\mathrm{i}}$, G. Vannoni ${ }^{\mathrm{x}}$, M. Vecchi ${ }^{\mathrm{b}}$, P. Vernin ${ }^{\mathrm{x}}$, E. Visser $^{\mathrm{h}}$, S. Wagner ${ }^{\mathrm{f}}, \mathrm{G}$. Wijnker ${ }^{\mathrm{h}}$, J. Wilms $^{\text {aa }}$, E. de Wolf ${ }^{\text {h,ac }}$, H. Yepes ${ }^{n}$, D. Zaborov ${ }^{\text {aj }}$, J.D. Zornoza ${ }^{n}$, J. Zúñiga ${ }^{\text {n }}$

a Institut d'Investigació per a la Gestió Integrada de les Zones Costaneres (IGIC) - Universitat Politècnica de València. C/ Paranimf 1, 46730 Gandia, Spain

${ }^{\mathrm{b}}$ CPPM, Aix-Marseille Université, CNRS/IN2P3, Marseille, France

${ }^{\mathrm{C}}$ GRPHE - Institut universitaire de technologie de Colmar, 34 rue du Grillenbreit, BP 50568 - 68008 Colmar, France

${ }^{\mathrm{d}}$ Technical University of Catalonia, Laboratory of Applied Bioacoustics, Rambla Exposició, 08800 Vilanova i la Geltrú, Barcelona, Spain

e INFN - Sezione di Genova, Via Dodecaneso 33, 16146 Genova, Italy

${ }^{\mathrm{f}}$ Friedrich-Alexander-Universität Erlangen-Nürnberg, Erlangen Centre for Astroparticle Physics, Erwin-Rommel-Str. 1, 91058 Erlangen, Germany

${ }^{\mathrm{g}}$ Direction des Sciences de la Matière - Institut de recherche sur les lois fondamentales de l'Univers - Service d'Electronique des Détecteurs et d'Informatique, CEA Saclay, 91191 Gif-sur-Yvette Cedex, France

${ }^{\mathrm{h}}$ Nikhef, Science Park, Amsterdam, The Netherlands

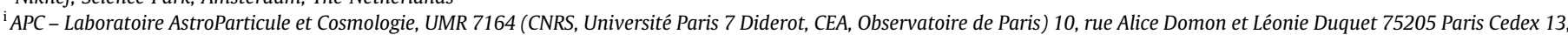
France

${ }^{\mathrm{j}}$ LAM - Laboratoire d'Astrophysique de Marseille, Pôle de l'Étoile Site de Château-Gombert, rue Frédéric Joliot-Curie 38, 13388 Marseille Cedex 13, France

${ }^{\mathrm{k}}$ INFN - Sezione di Bologna, Viale Berti-Pichat 6/2, 40127 Bologna, Italy

${ }^{1}$ Dipartimento di Fisica dell'Università, Viale Berti Pichat 6/2, 40127 Bologna, Italy

${ }^{\mathrm{m}}$ INFN - Sezione di Pisa, Largo B. Pontecorvo 3, 56127 Pisa, Italy

${ }^{\mathrm{n}}$ IFIC - Instituto de Física Corpuscular, Edificios Investigación de Paterna, CSIC - Universitat de València, Apdo. de Correos 22085, 46071 Valencia, Spain

${ }^{\circ}$ INFN - Sezione di Roma, P.le Aldo Moro 2, 00185 Roma, Italy

${ }^{\mathrm{p}}$ Dipartimento di Fisica dell'Università La Sapienza, P.le Aldo Moro 2, 00185 Roma, Italy

${ }^{\mathrm{q}}$ Clermont Université, Université Blaise Pascal, CNRS/IN2P3, Laboratoire de Physique Corpusculaire, BP 10448, 63000 Clermont-Ferrand, France

${ }^{\mathrm{r}}$ Géoazur - Université de Nice Sophia-Antipolis, CNRS/INSU, IRD, Observatoire de la Côte d'Azur and Université Pierre et Marie Curie, BP 48,06235 Villefranche-sur-mer, France

s INFN - Sezione di Bari, Via E. Orabona 4, 70126 Bari, Italy

${ }^{\mathrm{t}}$ COM - Centre d'Océanologie de Marseille, CNRS/INSU et Université de la Méditerranée, 163 Avenue de Luminy, Case 901, 13288 Marseille Cedex 9, France

uINFN - Laboratori Nazionali del Sud (LNS), Via S. Sofia 62, 95123 Catania, Italy

${ }^{\mathrm{v}}$ Univ Paris-Sud, 91405 Orsay Cedex, France

${ }^{\mathrm{w}}$ Kernfysisch Versneller Instituut (KVI), University of Groningen, Zernikelaan 25, 9747 AA Groningen, The Netherlands 


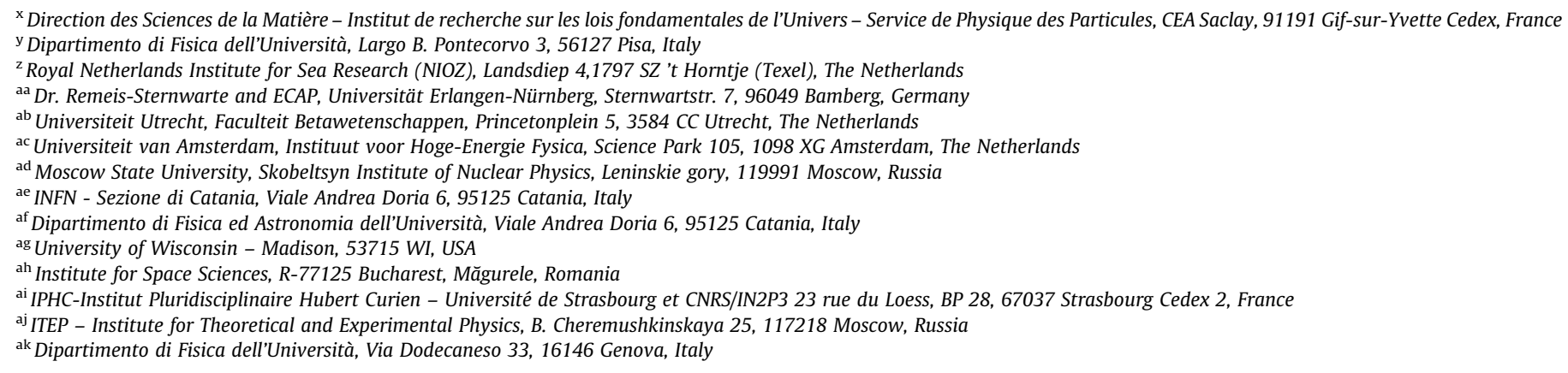

\section{A R T I C L E I N F O}

\section{Article history:}

Received 17 November 2011

Received in revised form 10 April 2012

Accepted 2 June 2012

Available online 9 June 2012

\section{Keywords:}

ANTARES

Neutrino astronomy

Fermi LAT transient sources

Time-dependent search

Blazars

\begin{abstract}
A B S T R A C T
The ANTARES telescope is well-suited to detect neutrinos produced in astrophysical transient sources as it can observe a full hemisphere of the sky at all times with a high duty cycle. Radio-loud active galactic nuclei with jets pointing almost directly towards the observer, the so-called blazars, are particularly attractive potential neutrino point sources. The all-sky monitor LAT on board the Fermi satellite probes the variability of any given gamma-ray bright blazar in the sky on time scales of hours to months. Assuming hadronic models, a strong correlation between the gamma-ray and the neutrino fluxes is expected. Selecting a narrow time window on the assumed neutrino production period can significantly reduce the background.

An unbinned method based on the minimization of a likelihood ratio was applied to a subsample of data collected in 2008 (61 days live time). By searching for neutrinos during the high state periods of the AGN light curve, the sensitivity to these sources was improved by about a factor of two with respect to a standard time-integrated point source search. First results on the search for neutrinos associated with ten bright and variable Fermi sources are presented.
\end{abstract}

(c) 2012 Elsevier B.V. All rights reserved.

\section{Introduction}

Neutrinos are unique messengers to study the high-energy universe as they are neutral and stable, interact weakly and therefore travel directly from their point of creation to the Earth without absorption. Neutrinos could play an important role in understanding the mechanisms of cosmic ray acceleration and their detection from a cosmic source would be a direct evidence of the presence of hadronic acceleration. The production of high-energy neutrinos has been proposed for several kinds of astrophysical sources, such as active galactic nuclei (AGN), gamma-ray bursters (GRB), supernova remnants and microquasars, in which the acceleration of hadrons may occur (see Ref. [1] for a review).

Flat-Spectrum Radio Quasars (FSRQs) and BL Lacs, classified as AGN blazars, exhibit relativistic jets pointing almost directly towards the Earth and are some of the most violent variable high energy phenomena in the Universe [2]. These sources are among the most likely sources of the observed ultra high energy cosmic rays. Blazars typically display spectra with enhanced emission over two energy ranges: the IR/X-ray and $\mathrm{MeV} / \mathrm{TeV}$ peaks. The lower energy peak is generally agreed to be the product of synchrotron radiation from accelerated electrons. However, the origin of the higher energy peak remains to be clarified. In leptonic models [3], inverse Compton scattering of synchrotron photons (or other ambient photons) by accelerated electrons generates this high energy emission. In hadronic models [4], MeV-TeV gamma-rays and high energy neutrinos are produced through hadronic interactions

\footnotetext{
* Corresponding author.

E-mail address: dornic@in2p3.fr (D. Dornic).

1 Also at University of Leiden, the Netherlands.

2 On leave at DESY, Platanenallee 6, D-15738 Zeuthen, Germany.

${ }^{3}$ Now at University of California - Irvine, 92697 CA, USA.

4 Deceased.
}

of the high energy cosmic rays with radiation or gas clouds surrounding the source. The gamma-ray light curves of bright blazars measured by the LAT instrument on board the Fermi satellite reveal important time variability on timescales of hours to several weeks, with intensities much larger than the typical flux of the source in its quiescent state [5]. The high state periods of the gamma-ray light curves are used as tracers of the most promising neutrino emission periods with the hypothesis of the time coincidence of both emissions.

This paper presents the results of the first time-dependent search for cosmic neutrino sources by the ANTARES telescope. The data sample used in this analysis and the comparison to Monte Carlo simulations are described in Section 2, together with a discussion on the systematic uncertainties. The point source search algorithm used in this time-dependent analysis is explained in Section 3. The search results are presented in Section 4 for ten selected candidate sources.

\section{ANTARES}

The ANTARES Collaboration completed the construction of a neutrino telescope in the Mediterranean Sea with the connection of its twelfth detector line in May 2008 [6]. The telescope is located $40 \mathrm{~km}$ off the Southern coast of France $\left(42^{\circ} 48^{\prime} \mathrm{N}, 6^{\circ} 10^{\prime} \mathrm{E}\right)$ at a depth of $2475 \mathrm{~m}$. It comprises a three-dimensional array of photomultipliers housed in glass spheres (optical modules [7]), distributed along twelve slender lines, $450 \mathrm{~m}$ height, anchored at the sea bottom and kept taut by a buoy at the top. The average distance between lines is $60 \mathrm{~m}$. Each line is composed of 25 storeys of triplets of optical modules (OMs), each housing one 10 -inch photomultiplier. The lines are subject to the sea currents and can change shape and orientation. A positioning system based on hydrophones, compasses and tiltmeters is used to monitor the detector geometry with an accuracy of $10 \mathrm{~cm}$. 
The main goal of the experiment is to search for high energy neutrinos with energies greater than $100 \mathrm{GeV}$ by detecting muons produced by the neutrino charged current interaction in the vicinity of the detector. Due to the large background from downgoing atmospheric muons, the telescope is optimised for the detection of upgoing muons as only they can originate from neutrinos.

Muons induce the emission of Cherenkov light in the sea water. The arrival time and intensity of the Cherenkov light on the OMs are digitised into hits and transmitted to shore. Events containing muons are selected from the continuous deep sea optical backgrounds due to natural radioactivity and bioluminescence. A detailed description of the detector and the data acquisition is given in [6,8].

The arrival times of the hits are calibrated as described in [9]. A L1 hit is defined either as a high-charge hit, or as hits separated by less than $20 \mathrm{~ns}$ on OMs of the same storey. At least five L1 hits are required throughout the detector within a time window of $2.2 \mu \mathrm{s}$, with the relative photon arrival times being compatible with the light coming from a relativistic particle. Independently, events which have L1 hits on two sets of adjacent or next-to-adjacent floors are also selected. This second condition mainly improves the trigger efficiency by 10-15\% at energy lower than $1 \mathrm{TeV}$.

The data used in this analysis were taken in the period from September 6 to December 31, 2008 (54720 to 54831 modified Julian days, MJD) with the twelve line detector. This period overlaps with the availability of the first data from the LAT instrument onboard the Fermi satellite. The corresponding effective live time is 60.8 days. Atmospheric neutrinos are the main source of background in the search for astrophysical neutrinos. These upgoing neutrinos are produced by the interaction of cosmic rays in the Earth's atmosphere. To account for this background, neutrino events were simulated according to the parametrisation of the atmospheric neutrino flux from Ref. [10] with 100 years equivalent live time. Only charged current interactions of muon neutrinos and antineutrinos were considered. An additional source of background is due to downgoing atmospheric muons mis-reconstructed as upgoing. Downgoing atmospheric muons were simulated with the MUPAGE package [11] with a sample equivalent to $1 / 10$ of the actual live time. In both cases, the same detector geometry is used and the Cherenkov light was propagated taking into account light absorption and scattering in sea water [12].

From the timing and position information of the hits, muon tracks are reconstructed using a multi-stage fitting procedure, based on Ref. [13]. The initial fitting stages provide the hit selection

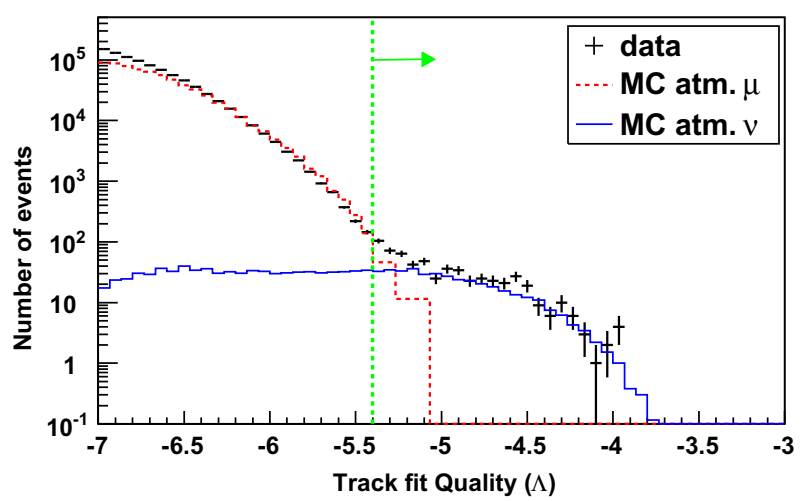

Fig. 1. Track fit quality $(\Lambda)$ distribution for upgoing events in data (dots) and Monte Carlo samples (atmospheric muons: dashed line; atmospheric neutrinos: continuous line). Events are selected with an error estimate lower than 1 degree. The green dashed vertical line corresponds to the optimised event selection $(\Lambda>-5.4)$. (For interpretation of the references to colour in this figure legend, the reader is referred to the web version of this article.) and starting point for the final fit. The final stage consists of a maximum likelihood fit of the observed hit times and includes the contribution of optical background hits.

To discard most of the downgoing atmospheric muon background, a cut on the zenith angle $\left(\theta>90^{\circ}\right)$ is applied to select only upgoing events. To suppress the remaining atmospheric muons mis-reconstructed as upgoing, tracks are also required to have a good reconstruction quality. The latter is quantified by a parameter, $\Lambda$, which is based on the value of the likelihood function obtained for the fitted muon (see Ref. [13] for details). The cumulative distribution of $\Lambda$ for muons reconstructed as upgoing is shown in Fig. 1 along with the simulated contributions from atmospheric muons and neutrinos. The small discrepancy between data and simulation at very negative values of lambda is due to pure optical noise ( $40 \mathrm{~K}$ decay and bioluminescence) background events not taken into account in the Monte Carlo simulations. The error estimate of the reconstructed track direction obtained from the track fit is required to be smaller than 1 degree. This error is extracted from the diagonal terms of the covariance error matrix of the track fit. Fig. 2 shows the simulated distributions of the error estimate parameter for both atmospheric muon and neutrinos.

For this analysis, events are selected with $\Lambda>-5.4$. This value yields to the best $5 \sigma$ discovery potential and results from the optimal compromise between the atmospheric neutrino and muon background reduction and the efficiency of the cosmic neutrino signal with an assumed spectrum proportional to $E_{v}^{-2}$, where $E_{v}$ is the neutrino energy. The final sample consists of 628 events obtained in 60.8 days. The simulations indicate that the selected sample contains $60 \%$ atmospheric neutrinos; the rest being misreconstructed atmospheric muons. The average energy (5-95\%) is $70 \mathrm{GeV}-11 \mathrm{TeV}$ for atmospheric neutrinos and $2 \mathrm{TeV}-1.4 \mathrm{PeV}$ for cosmic neutrinos with a $E_{v}^{-2}$ spectrum.

The angular resolution of the reconstructed neutrino direction cannot be determined directly from the data and has to be estimated from simulation. Comparison of data and Monte Carlo in which the time accuracy of the hits was degraded by up to $3 \mathrm{~ns}$ constrains the systematic uncertainty of the angular resolution to about $0.1^{\circ}[14]$. This uncertainty incorporates effects on possible detector mis-alignments, inaccuracies in the simulation of light propagation in the water or the time response distribution of the PMT. Fig. 3 shows the cumulative distribution of the angular difference between the reconstructed muon direction and the neutrino direction for an assumed spectrum proportional to $E_{v}^{-2}$. For the

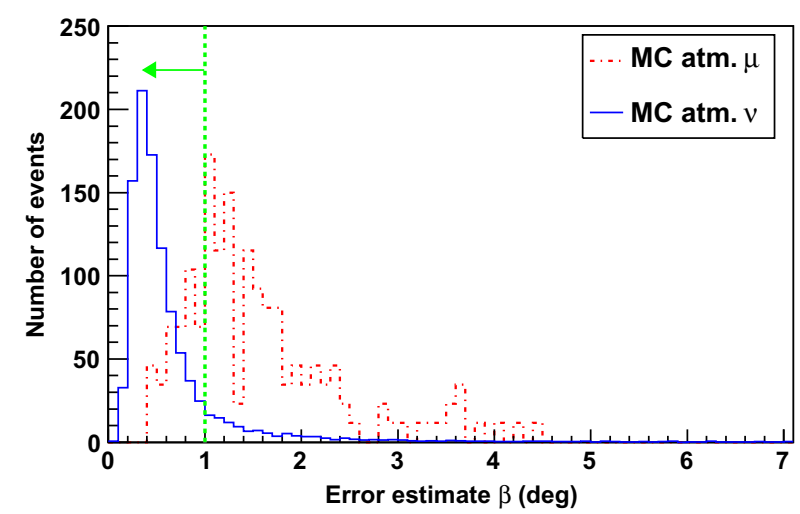

Fig. 2. Distribution of the error estimate on the direction of the reconstructed muon track for upward reconstructed simulated atmospheric muons (dashed line) and atmospheric neutrinos (continuous line). Events are selected with $\Lambda>-5.4$. The green dashed vertical line corresponds to the event selection $\left(\beta<1^{\circ}\right.$ ). (For interpretation of the references to colour in this figure legend, the reader is referred to the web version of this article.) 


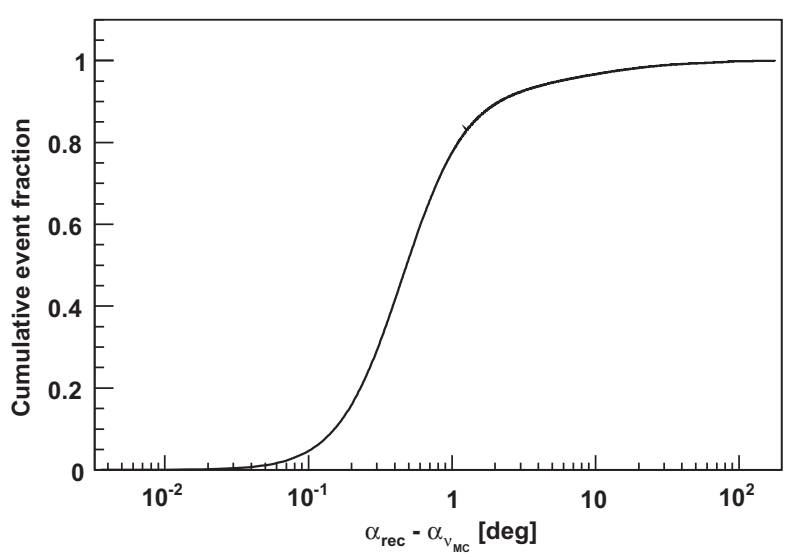

Fig. 3. Cumulative distribution of the angle between the true Monte Carlo neutrino direction $\left(\alpha_{v_{M C}}\right)$ and the reconstructed muon direction $\left(\alpha_{\text {rec }}\right)$ for an $E_{v}^{-2}$ flux of upgoing neutrino events selected for this analysis.

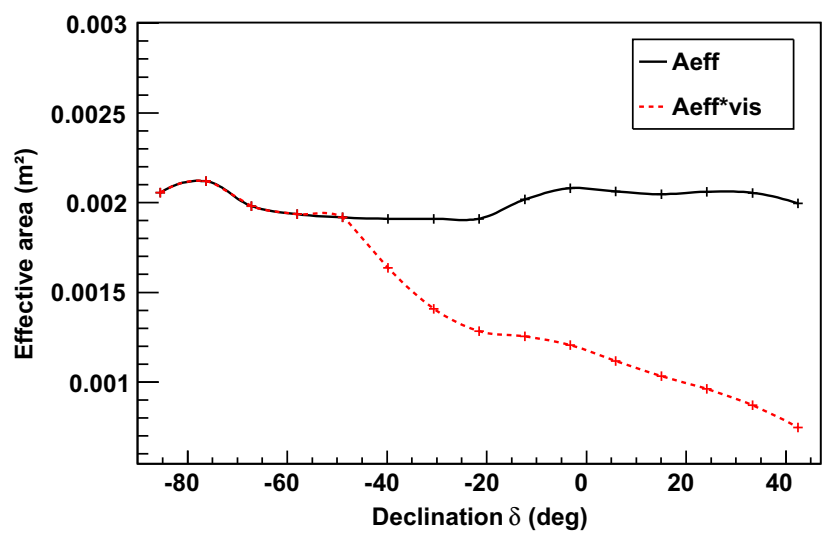

Fig. 4. ANTARES muon neutrino and antineutrino effective area (continuous line) as a function of the declination of the source computed from the Monte Carlo simulation for an $\mathrm{E}_{v}^{-2}$ flux of upgoing muons selected for this analysis. The product of the effective area by the visibility (i.e. fraction of the time the source is visible at the ANTARES location) is shown with the dashed line.

considered period, the median resolution is estimated to be $0.5 \pm$ 0.1 degrees.

The effective area for muon neutrinos is defined as the ratio between the rate of selected neutrino events and the cosmic neutrino flux. Fig. 4 shows the muon neutrino and antineutrino effective area of the ANTARES telescope as a function of the declination of the source, after integrating over the energy with an assumed spectrum proportional to $E_{v}^{-2}$ between $10 \mathrm{GeV}$ and $10 \mathrm{PeV}$. In the flux limits (see Section 4), a conservative uncertainty on the detection efficiency of about $30 \%$ is taken into account. This number includes contributions on the uncertainty of the sea water optical parameters [12] and the OM properties such as efficiency and angular acceptance.

\section{Time-dependent search algorithm}

The time-dependent point source analysis is performed using an unbinned method based on a likelihood ratio maximisation. The data are parametrised as a mixture of cosmic neutrino signal and atmospheric neutrino and muon background. The goal is to determine, at a given point in the sky and at a given time, the relative contribution of each component and to calculate the proba- bility to have a signal above background in a given model. The likelihood ratio, $\lambda$, is the logarithm of the ratio of the probability density for the hypothesis of signal and background $\left(H_{\text {sig }+b k g}\right)$ over the probability density of only background $\left(H_{b k g}\right)$ :

$\lambda=\sum_{i=1}^{N} \log \frac{P\left(x_{i} \mid H_{\text {sig }+b k g}\right)}{P\left(x_{i} \mid H_{b k g}\right)}=\sum_{i=1}^{N} \log \frac{\frac{n_{\text {sig }}}{N} P_{\text {sig }}\left(\alpha_{i}, t_{i}\right)+\left(1-\frac{n_{\text {sig }}}{N}\right) P_{b k g}\left(\delta_{i}, t_{i}\right)}{P_{b k g}\left(\alpha_{i}, t_{i}\right)}$

where $n_{\text {sig }}$ is the unknown number of signal events determined by the fit and $\mathrm{N}$ is the total number of events in the considered data sample. $P_{s i g}\left(\alpha_{i}, t_{i}\right)$ and $P_{b k g}\left(\delta_{i}, t_{i}\right)$ are the probability density functions (PDF) for signal and background respectively. For a given event $i, t_{i}, \delta_{i}$ and $\alpha_{i}$ represent the time of the event, its declination and the angular separation from the source under consideration.

The probability densities $P_{s i g}$ and $P_{b k g}$ are factorised into a purely directional and a purely time-related component. For signal events, the directional PDF is described by the one dimensional point spread function (PSF), which is the probability density of reconstructing an event at an angular distance $\alpha$ from the true source position. The shape of the time PDF for the signal event is extracted directly from the gamma-ray light curve assuming proportionality between the gamma-ray and the neutrino fluxes. The directional and time PDF for the background are derived from the data using the observed declination distribution of the selected events and the observed one-day binned time distribution of all the reconstructed muons respectively. Fig. 5 shows the time distribution of all the reconstructed events and the selected upgoing events for this analysis. Once normalised to an integral equal to 1 , the distribution for all reconstructed events is used directly as the time PDF for the background. Empty bins in the histograms correspond to periods with no data taking (i.e. detector in maintenance) or with very poor quality data (high bioluminescence or bad calibration).

The statistical interpretation of the search result relies on simulated pseudo experiments (PE) in which the background events are randomly generated by sampling the declination and the time from the parametrisation $P_{b k g}\left(\delta_{i}, t_{i}\right)$ and the right ascension from a uniform distribution. Events from a neutrino point source are simulated by adding events around the desired coordinates according to the point spread function and the time distribution of the studied source. Systematic uncertainties (cf Section 2) are incorporated directly into the pseudo experiment generation. The uncertainties on the angular resolution and orientation of the detector were incorporated by varying the simulated characteristics of the signal

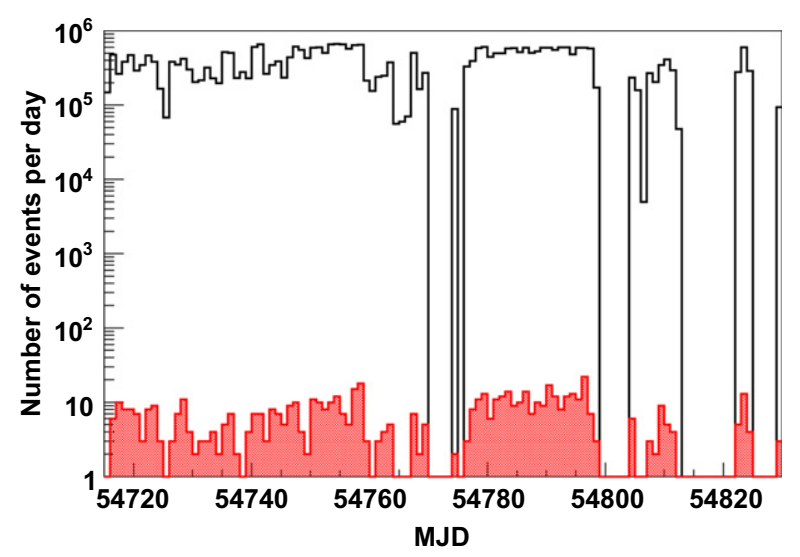

Fig. 5. Time distribution of the reconstructed events. Upper histogram (black line): distribution of all reconstructed events. Bottom filled histogram (red): distribution of selected upgoing events. (For interpretation of the references to colour in this figure legend, the reader is referred to the web version of this article.) 


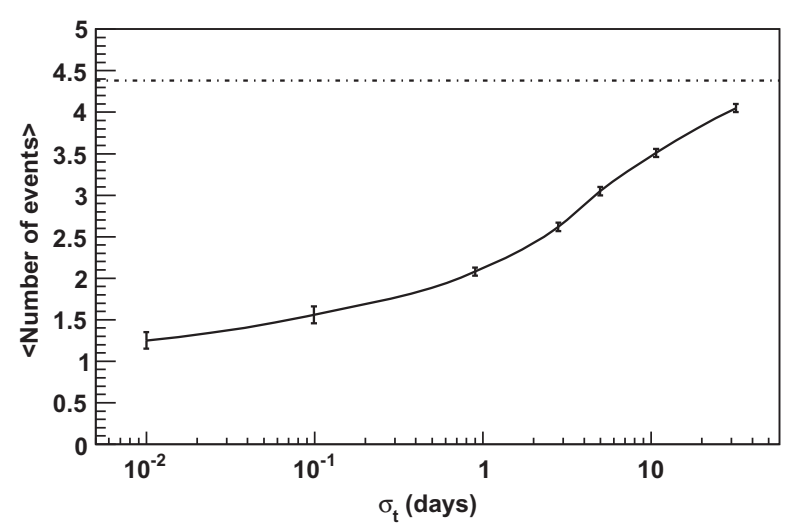

Fig. 6. Average number of events (solid line) required for a $5 \sigma$ discovery ( $50 \%$ probability) from a single source of fixed fluence located at a declination of $-40^{\circ}$ as a function of the width of the flare period $\left(\sigma_{t}\right)$ for the 60.8 day analysis. These numbers are compared to that obtained without using the timing information (dashed line).

events within the assigned range of $0.1^{\circ}$. The uncertainties affecting the effective area is accounted for in the limits by varying the number of injected signal events by a Gaussian error with a width of $30 \%$.

The null hypothesis corresponds to $n_{\text {sig }}=0$. The obtained value of $\lambda_{\text {data }}$ on the data is then compared to the distribution of $\lambda\left(n_{\text {sig }}=0\right)$. Large values of $\lambda_{\text {data }}$ compared to the distribution of $\lambda\left(n_{\text {sig }}=0\right)$ reject the null hypothesis with a confidence level (C.L.) equal to the fraction of the number of PE above $\lambda_{\text {data }}$. The fraction of PE for which $\lambda\left(n_{\text {sig }}=0\right)$ is above $\lambda_{\text {data }}$ is referred to as the $p$-value. The discovery potential is then defined as the average number of signal events required to achieve a $p$-value lower than $5 \sigma$ in $50 \%$ of the PEs. In the same way, the sensitivity is defined as the average signal required to obtain a $p$-value less than that of the median of the $\lambda\left(n_{\text {sig }}=0\right)$ distribution in $90 \%$ of the PEs. In the absence of evidence of a signal, an upper limit on the neutrino fluence is obtained and defined as the integral in energy and time of the flux upper limit with an assumed energy spectrum proportional to $E_{v}^{-2}$ from $10 \mathrm{GeV}$ to $10 \mathrm{PeV}$. The limits are calculated according to the classical (frequentist) method for upper limits [15].

The performance of the time-dependent analysis was computed by applying this unbinned algorithm for a single source assuming a single square-shape flare of fixed fluence with a width varying from 0.01 days to 32 days. The solid line in Fig. 6 shows the average number of events required for a discovery from one source located at a declination of $-40^{\circ}$ as a function of the width of the flare. The numbers in the black line are compared to that obtained without using the timing information (dashed line). The time-integrated analysis was performed using the same inputs (data sample, source characteristics) and the same unbinned algorithm but with the time PDF term set to 1 . Time dependent searches have their power by cutting down on the atmospheric neutrino and muon background, shorter flares cut more background. The flare timing information yields an improvement of the discovery potential by about a factor 2 (1.3) with respect to a standard time-integrated point source search [14] when looking for a 1 (10) day flare.

\section{Search for neutrino emission from gamma-ray flares}

The time-dependent analysis was applied to bright and variable Fermi blazar sources reported in the first-year Fermi LAT catalogue [16] and in the LBAS catalogue (LAT Bright AGN sample [17]). Sources were selected in the sky visible to ANTARES and that had at least one day binned gamma-ray flux in the high state periods greater than
Table 1

List of bright variable Fermi blazars selected for this analysis [17].

\begin{tabular}{lllccl}
\hline Name & OFGL name & Class & RA [ $\left.{ }^{\circ}\right]$ & Dec $\left[{ }^{\circ}\right]$ & Redshift \\
\hline PKS0208-512 & J0210.8-5100 & FSRQ & 32.70 & -51.2 & 1.003 \\
AO0235+164 & J0238.6 +1636 & BLLac & 39.65 & 16.61 & 0.940 \\
PKS0454-234 & J0457.1-2325 & FSRQ & 74.28 & -23.43 & 1.003 \\
OJ287 & J0855.4+2009 & BLLac & 133.85 & 20.09 & 0.306 \\
WComae & J1221.7+28.14 & BLLAc & 185.43 & 28.14 & 0.102 \\
3C273 & J1229.1+0202 & FSRQ & 187.28 & 2.05 & 0.158 \\
3C279 & J1256.1-0548 & FSRQ & 194.03 & -5.8 & 0.536 \\
PKS1510-089 & J1512.7-0905 & FSRQ & 228.18 & -9.09 & 0.36 \\
3C454.3 & J2254.0+1609 & FSRQ & 343.50 & 16.15 & 0.859 \\
PKS2155-304 & J2158.8-3014 & BLLac & 329.70 & -30.24 & 0.116 \\
\hline
\end{tabular}

$8 \times 10^{-7}$ photons $\mathrm{cm}^{-2} \mathrm{~s}^{-1}$ above $100 \mathrm{MeV}$ and showed significant time variability on time scales of days to weeks in the studied time period. A source is assumed variable in the LBAS catalogue when the observation has a probability of less than $1 \%$ of being a steady source. This list includes six Flat-Spectrum Radio Quasars and four BL-Lacs. Only four bright and nearby sources in the considered sample, PKS2155-304 [18], PKS1510-089 [19], 3C279 [20] and WComae [21], have been detected by the ground Cherenkov telescopes HESS, MAGIC or VERITAS.

Table 1 lists the characteristics of the ten selected sources.

The light curves published on the Fermi web page for the monitored sources [22] are used for this analysis. They correspond to the time evolution of the daily gamma-ray flux in the band 0.1-300 GeV since August 2008. The high state periods are defined using a simple and robust method based on three main steps. Firstly, the baseline is determined with an iterative linear fit. After each fit, bins more than two sigma $\left(\sigma_{B L}\right)$ above the baseline $(\mathrm{BL})$ are removed. Secondly, seeds for the high state periods are identified by searching for bins significantly above the baseline according to the criteria:

$\left(F-\sigma_{F}\right)>\left(B L+2 * \sigma_{B L}\right)$ and $F>\left(B L+3 * \sigma_{B L}\right)$

where $\mathrm{F}$ and $\sigma_{F}$ represent the flux and the uncertainty on this flux for each bin, respectively. For each seed, the adjacent bins for which the emission is compatible with the flare are added if they satisfy: $\left(F-\sigma_{F}\right)>\left(B L+\sigma_{B L}\right)$. Finally, an additional delay of 0.5 days is added before and after the flare in order to take into account that the precise time of the flare is not known (1-day binned light curve). With this definition, a flare has a width of at least two days. The flare search was performed on the 2008-2010 Fermi light curve

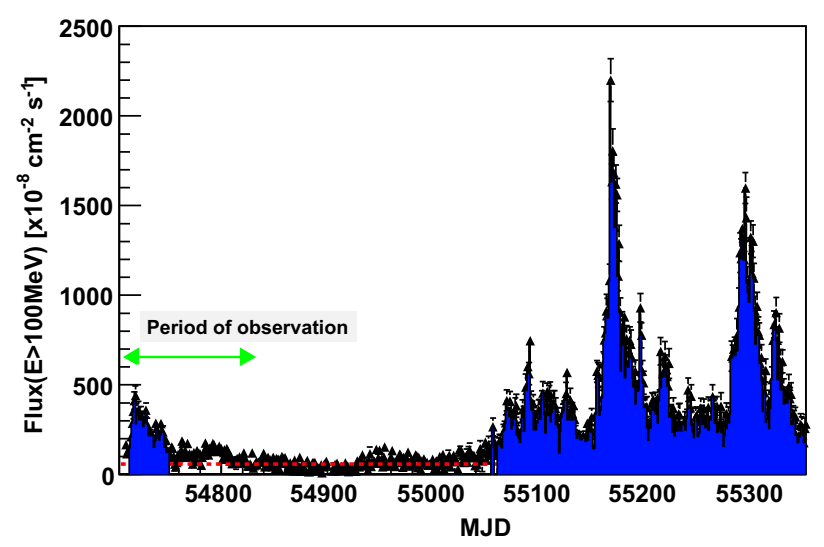

Fig. 7. Gamma-ray light curve (black points) of the blazar 3C454.3 measured by the LAT instrument onboard the Fermi satellite above $100 \mathrm{MeV}$ for almost two years of data. The shaded histogram (blue) indicates the high state periods. The dashed line (red) represents the fitted baseline. (For interpretation of the references to colour in this figure legend, the reader is referred to the web version of this article.) 
Table 2

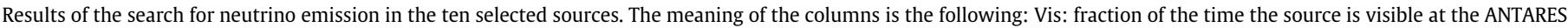

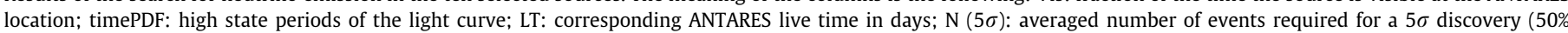


including the systematic errors.

\begin{tabular}{|c|c|c|c|c|c|c|}
\hline Source & Vis & timePDF (MJD-54000) & LT & $\mathrm{N}(5 \sigma)$ & $N_{o b s}$ & Fluence U.L \\
\hline PKS0208-512 & 1.0 & $\begin{array}{l}712-5,722-4,745-7, \\
750-2,753-7,764-74, \\
820-2\end{array}$ & 8.8 & 4.5 & 0 & 2.8 \\
\hline A00235 + 164 & 0.41 & $\begin{array}{l}710-33,738-43,746-64, \\
766-74,785-7,805-8, \\
810-2\end{array}$ & 24.5 & 4.3 & 0 & 18.7 \\
\hline PKS1510-089 & 0.55 & $\begin{array}{l}716-9,720-5,726-35 \\
788-90,801-3\end{array}$ & 4.9 & 3.8 & 0 & 2.8 \\
\hline $3 C 273$ & 0.49 & $714-6,716-8,742-5$ & 2.4 & 2.5 & 0 & 1.1 \\
\hline $3 C 279$ & 0.53 & $\begin{array}{l}749-51,787-809 \\
812-5,817-21,824-6\end{array}$ & 13.8 & 5.0 & 1 & 8.2 \\
\hline $3 C 454.3$ & 0.41 & $\begin{array}{l}713-51,761-5,767-9 \\
784-801\end{array}$ & 30.8 & 4.4 & 0 & 23.5 \\
\hline OJ287 & 0.39 & $\begin{array}{l}733-5,752-4,760-2, \\
768-70,774-6,800-2, \\
814-6\end{array}$ & 4.3 & 3.9 & 0 & 3.4 \\
\hline PKS0454-234 & 0.63 & $743-5,792-6,811-3$ & 6.0 & 3.3 & 0 & 2.9 \\
\hline WComae & 0.33 & $\begin{array}{l}726-9,771-3,790-2 \\
795-7,815-7\end{array}$ & 3.9 & 3.8 & 0 & 3.6 \\
\hline PKS2155-304 & 0.68 & $\begin{array}{l}753-5,766-8,799-801 \\
828-30\end{array}$ & 3.1 & 3.7 & 0 & 1.6 \\
\hline
\end{tabular}

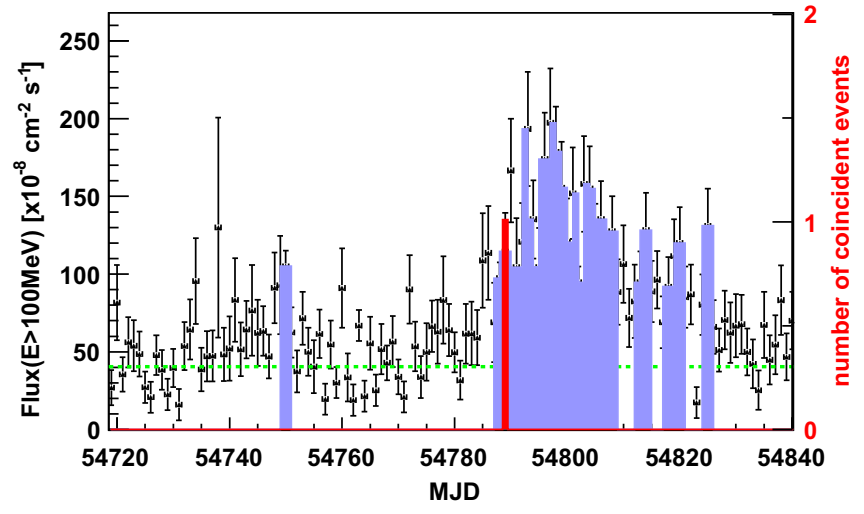

Fig. 8. Gamma-ray light curve (dots) of the blazar 3C279 measured by the LAT instrument onboard the Fermi satellite above $100 \mathrm{MeV}$. The light shaded histogram (blue) indicates the high state periods. The dashed line (green) corresponds to the fitted baseline. The red histogram displays the time of the associated ANTARES neutrino event. (For interpretation of the references to colour in this figure legend, the reader is referred to the web version of this article.)

and then the list of flare periods was restrained to the studied period. This procedure takes into account the errors on the gamma-ray data points and without any interpolation that would smear out the signal in time and make the analysis less effective. Moreover, it allows finding the minimal threshold to apply to the gamma-ray flux to identify periods statistically inconsistent with a steady emission. Fig. 7 shows the time distribution of the Fermi LAT gamma-ray light curve of 3C454.3 for almost two years of data and the corresponding selected high state periods. With the hypothesis that the neutrino emission follows the gamma-ray emission, the signal time PDF is simply the normalised light curve of only the high state periods. The third column of Table 2 lists the flaring periods for the ten sources found from September to December 2008.

The results of the search for coincidences between flares and neutrinos are listed in Table 2. For nine sources, no coincidences are found. For 3C279, a single high-energy neutrino event is found in spatial and time coincidence during a large flare in November 2008. Fig. 8 shows the time distribution of the Fermi gamma-ray light curve of 3C279 and the time of the coincident neutrino event. This event was reconstructed with 89 hits distributed on ten lines with a track fit quality $\Lambda=-4.4$. The particle track direction is reconstructed at $0.56^{\circ}$ from the source location. The pre-trial $p$-value is $1.0 \%$. However, the post-trial probability computed taking into account the ten searches is $10 \%$; this occurrence is thus compatible with a background fluctuation. In the absence of a discovery, upper limits on the neutrino fluence were computed and are shown including the systematic errors in the last column of Table 2 . The overall effect of including the systematic uncertainties is a degradation of the limit by $10 \%$.

\section{Summary}

This paper presents the first time-dependent search for cosmic neutrinos using the data taken with the full twelve line ANTARES detector during the last four months of 2008. For short variable sources, time-dependent point searches are more sensitive than time-integrated searches due to the large reduction of the background assuming that neutrinos and gamma-rays are emitted in the same periods. This search was applied to ten very bright and variable Fermi LAT blazars. One neutrino event was detected in time/direction coincidence with the gamma-ray emission in only one case, for a flare of 3C279 in November 2008, with a post-trial probability of $10 \%$. Upper limits were obtained on the neutrino fluence for the ten selected sources.

\section{Acknowledgments}

The authors acknowledge the financial support of the funding agencies: Centre National de la Recherche Scientifique (CNRS), Commissariat à l'énergie atomique et aux énergies alternatives (CEA), Agence National de la Recherche (ANR), Commission Européenne (FEDER fund and Marie Curie Program), Région Alsace (contrat CPER), Région Provence-Alpes-Côte d'Azur, Département du Var and Ville de La Seyne-sur-Mer, France; Bundesministerium für Bildung und Forschung (BMBF), Germany; Istituto Nazionale di Fisica Nucleare (INFN), Italy; Stichting voor Fundamenteel Onderzoek der Materie (FOM), Nederlandse organisatie voor Wetenschappelijk Onderzoek (NWO), the Netherlands; Council of 
the President of the Russian Federation for young scientists and leading scientific schools supporting grants, Russia; National Authority for Scientific Research (ANCS), Romania; Ministerio de Ciencia e Innovación (MICINN), Prometeo of Generalitat Valenciana and MultiDark, Spain. We also acknowledge the technical support of Ifremer, AIM and Foselev Marine for the sea operation and the CC-IN2P3 for the computing facilities.

\section{References}

[1] J.K. Becker, Phys. Rep. 458 (2008) 173.

[2] C.M. Urry, P. Padovani, PASP 107 (1995) 803.

[3] S.D. Bloom, A.P. Marscher, Astrophys. J. 461 (1996) 657; L. Maraschi, G. Ghisellini, A. Celotti, Astrophys. J. 397 (1992) L5 C.D. Dermer, R. Schlickeiser, Astrophys. J. 416 (1993) 458.

[4] T.K. Gaisser, F. Halzen, T. Stanev, Phys. Rep. 258 (1995) 173; J.G. Learned, K. Mannheim, Ann. Rev. Nucl. Part. Sci. 50 (2000) 679; C.D. Dermer, A. Atoyan, Phys. Rev. Lett. 91 (2003) 071102

M. Böttcher, Astrophys. Space Sci. 309 (2007) 95;

F.A. Aharonian, New Astron. 5 (2000) 377.
[5] A.A. Abdo et al., ApJ 722 (2010) 520.

[6] M. Ageron et al., Nucl. Instrum. Methods A656 (2011) 11.

[7] P. Amram et al., Nucl. Instrum. Methods A484 (2002) 369.

[8] J.A. Aguilar et al., Nucl. Instrum. Methods A570 (2007) 107.

[9] J.A. Aguilar et al., Astropart. Phys. 34 (2011) 539.

[10] V. Agrawal, T.K. Gaisser, P. Lipari, T. Stanev, Phys. Rev. D53 (1996) 1314.

[11] Y. Becherini, A. Margiotta, M. Sioli, M. Spurio, Astropart. Phys. 25 (2006) 1; G. Carminati, M. Bazzotti, A. Margiotta, M. Spurio, Comput. Phys. Commun. 179 (2008) 915.

[12] J.A. Aguilar et al., Astropart. Phys. 23 (2005) 131.

[13] Heijboer, A., Ph.D. Thesis, Universiteit van Amsterdam, Amsterdam, The Netherlands. <http://antares.in2p3.fr/Publications/thesis/2004/Aart-Heijboerphd.pdf>.

[14] Adrián-Martínez, S. et al., Astrophys. J., accepted. <arXiv:1108.0292v1[astroph.HE]>.

[15] J. Neyman, Philos. Trans. Royal Soc. London, Series A 236 (1937) 333.

[16] A.A. Abdo et al., Astophys. J. Suppl. 188 (2010) 405.

[17] A.A. Abdo et al., Astrophys. J. 715 (2010) 429.

[18] F. Aharonian et al., A\&A 430 (2005) 865-875.

[19] S. Wagner et al., HEAD 27 (2010) 06.

[20] J. Albert et al., Science 320 (2008) 1752.

[21] V.A. Acciari et al., Astrophys. J. 684 (2008) 73-77.

[22] Monitored source list, <http://fermi.gsfc.nasa.gov/ssc/data/access/lat/>. 